\title{
PHF6 Deletions May Cause Borjeson-Forssman-Lehmann Syndrome in Females
}

\author{
S. Berland ${ }^{a, b} \quad$ K. Alme $e^{a, c} \quad$ A. Brendehaug ${ }^{a} \quad$ G. Houge ${ }^{a, d} \quad$ R. Hovland ${ }^{a, c}$ \\ ${ }^{a}$ Center for Medical Genetics and Molecular Medicine, Haukeland University Hospital, Bergen, bSection of \\ Clinical Genetics, Department of Pathology, St. Olavs Hospital, Trondheim, 'Department of Molecular Biology and \\ ${ }^{\mathrm{d} D e p a r t m e n t}$ of Clinical Medicine, University of Bergen, Bergen, Norway
}

\section{Key Words}

Female $\cdot$ Mental retardation $\cdot$ Oligonucleotide array analysis $\cdot$ PHF6 $\cdot$ Pigmentation disorders · Syndactyly • $\mathrm{X}$ chromosome inactivation

\begin{abstract}
In a 16-year-old girl with intellectual disability, irregular teeth, slight body asymmetry, and striated skin pigmentation, highly skewed X-inactivation increased the likelihood of an X-linked cause of her condition. Among these, prominent supraorbital ridges and hearing loss suggested a filaminopathy, but no filamin A mutation was found. The correct diagnosis, Borjeson-Forssman-Lehmann syndrome (BFLS, MIM\#301900), was first made when a copy number array identified a de novo $15-\mathrm{kb}$ deletion of the terminal 3 exons of the PHF6 gene. In retrospect, her phenotype resembled that of males with BFLS. Such deletions of PHF6 have not been reported previously. This might be because PHF6 mutations are rarely looked for in females since classical BFLS so far has been thought to be a male-specific syndrome, and large PHF6 deletions might be incompatible with male fetal survival. If this is the case, sporadic BFLS could be more frequent in females than in males.
\end{abstract}

Copyright $\odot 2011$ S. Karger AG, Basel
Borjeson-Forssman-Lehman syndrome (BFLS) is a rare $\mathrm{X}$-linked condition usually affecting males, with no or mild symptoms in female carriers. The condition was described in 1962 [Borjeson et al., 1962] and is caused by mutations in PHF6 [Lower et al., 2002]. To date, about 21 families or sporadic cases have been reported, resulting in a total of approximately 50 patients [Borjeson et al., 1962; Ardinger et al., 1984; Baumstark et al., 2003; Birrell et al., 2003; Lower et al., 2004; Turner et al., 2004; Vallee et al., 2004; Crawford et al., 2006; Carter et al., 2009; de Winter et al., 2009; Mangelsdorf et al., 2009; Chao et al., 2010]. Thirteen different mutations have been identified so far [Gecz et al., 2006; Mangelsdorf et al., 2009]: 8 missense mutations, 3 were probably truncating, 1 in-frame deletion [Lower et al., 2002; Baumstark et al., 2003; Crawford et al., 2006], and 1 affected splicing [Vallee et al., 2004]. The mutations were spread all over the gene, suggesting a loss-of-function mechanism.

PHF6 is thought to play a role in transcriptional regulation, cell growth, and proliferation [Gecz et al., 2006; Voss et al., 2007; Dephoure et al., 2008]. The protein contains 4 nuclear localization signals and 2 plant homeodomain (PHD)-like zinc fingers [Lower et al., 2002]. There are at least 3 mRNA splice variants. PHF6 expression is highest in the fetal brain, but marked expression can also be seen in other developing tissues [Voss et al., 2007]. In adults, the expression is highest in thymus, ovary, and

\section{KARGER \\ Fax +4161306 1234 E-Mail karger@karger.ch} www.karger.com
Center for Medical Genetics and Molecular Medicine

Haukeland University Hospital

NO-5021 Bergen (Norway)

Tel. +47 5597 5475, E-Mail siren.berland @ helse-bergen.no 
Table 1. Clinical findings in BFLS males and female carriers of PHF6 mutations

\begin{tabular}{|c|c|c|c|c|c|c|c|c|}
\hline \multirow[t]{2}{*}{ Features } & \multirow{2}{*}{$\begin{array}{l}\text { Our } \\
\text { case }\end{array}$} & \multicolumn{5}{|c|}{ Female $P H F 6$ mutation carriers from the literature ${ }^{a}$} & \multirow[t]{2}{*}{ Sum females } & \multirow[t]{2}{*}{ Sum males ${ }^{\mathrm{b}}$} \\
\hline & & $\mathrm{A}, \mathrm{n}=1$ & $B, n=4$ & $C, n=2$ & $\mathrm{D}, \mathrm{n}=4$ & $\mathrm{E}, \mathrm{n}=14$ & & \\
\hline Coarse face & $1 / 1$ & $1 / 1$ & $1 / 4$ & $2 / 2$ & $0 / 4$ & $2 / 14$ & $29 \%(n=24)$ & $92 \%(\mathrm{n}=24)$ \\
\hline Deep-set eyes & $1 / 1$ & $1 / 1$ & $0 / 3$ & $2 / 2$ & $0 / 4$ & $2 / 14$ & $24 \%(n=25)$ & $100 \%(\mathrm{n}=39)$ \\
\hline Large ears & $0 / 1$ & $1 / 1$ & $0 / 3$ & $2 / 2$ & $0 / 4$ & $11 / 14$ & $56 \%(n=25)$ & $100 \%(n=42)$ \\
\hline Obesity & $0 / 1$ & $1 / 1$ & $1 / 4$ & $1 / 2$ & nd & nd & $3 / 8$ & $68-77 \%(n=22 / 26)$ \\
\hline \multicolumn{9}{|l|}{$\begin{array}{l}\text { Oligomenorrhea/hypogo- } \\
\text { nadism and/or small }\end{array}$} \\
\hline external genitalia & $1 / 1$ & $1 / 1 ?$ & nd & nd & nd & $1 / 14$ & $19 \%(n=16)$ & $78-92 \%(n=18 / 25)$ \\
\hline Gynecomastia & - & - & - & - & - & - & - & $97-100 \%(\mathrm{n}=22 / 39)$ \\
\hline Tapering fingers & $1 / 1$ & nd & $1 / 4$ & $1 / 2$ & nd & $4 / 14$ & $33 \%(n=21)$ & $\sim 100 \%$ \\
\hline Syndactyly of toes & $1 / 1$ & $0 / 1 ?$ & $0 / 4$ & $0 / 2$ & $0 / 4$ & $0 / 14$ & $4 \%(n=26)$ & nd \\
\hline \multicolumn{9}{|l|}{ Short/broad toes/wide } \\
\hline sandal gap & $1 / 1$ & $1 / 1$ & $1 / 4$ & $1 / 2$ & nd & $6 / 14$ & $45 \%(n=22)$ & $\sim 100 \%$ \\
\hline \multicolumn{9}{|l|}{ Intellectual or learning dis- } \\
\hline ability & $1 / 1$ & $1 / 1$ & $1 / 4$ & $2 / 2$ & $0 / 4$ & $2 / 14$ & $27 \%(\mathrm{n}=26)$ & $100 \%(n=43)$ \\
\hline Skewed $\mathrm{X}$-inactivation $>90 \%$ & $1 / 1$ & $2 / 3^{c}$ & nd & $1 / 2$ & $0 / 4$ & $20 / 21^{\mathrm{c}}$ & $77 \%(\mathrm{n}=31)$ & - \\
\hline Short stature & $0 / 1$ & $0 / 1$ & $0 / 4$ & $0 / 2$ & nd & $1 / 14$ & $4.5 \%(\mathrm{n}=22)$ & $35-50 \%(n=22 / 37)$ \\
\hline Seizures & $0 / 1$ & $0 / 1$ & $0 / 4$ & $0 / 2$ & $0 / 4$ & $2 / 14$ & $7.5 \%(n=26)$ & $8-29 \%(n=21 / 39)$ \\
\hline Dental abnormalities & $1 / 1$ & nd & nd & nd & nd & nd & 1 patient reported & 2 patients reported \\
\hline Hearing loss & $1 / 1$ & nd & nd & nd & nd & nd & 1 patient reported & 1 patient reported \\
\hline Cancer & $0 / 1$ & $0 / 1$ & nd & $0 / 2$ & $0 / 4$ & nd & 0 patients reported & 2 patients reported \\
\hline \multicolumn{9}{|c|}{$\begin{array}{l}\text { The extent of clinical features in females is biased as unaf- } \\
\text { fected females are inconsistently reported, i.e. clinical data/pic- } \\
\text { tures are often lacking. nd = Insufficient or no data available. } \\
\text { a A = Crawford et al. [2005]; B = Mangelsdorf et al. [2009]; C = } \\
\text { Carter et al. [2008]; D = Baumstark et al. [2003]; E = Turner et al. } \\
\text { [2004]. }\end{array}$} \\
\hline
\end{tabular}

thyroid, with moderate expression in spleen, testes, and adipocytes [Van Vlierberghe et al., 2011].

Since almost all females with PHF6 mutations have been ascertained through family studies and since these females have mild or no symptoms [Gecz et al., 2006], BFLS is clinically conceived as an X-linked condition affecting males. The only reported exception was a 14-yearold girl with a de novo PHF6 frame-shift mutation and a rather mild phenotype [Crawford et al., 2006]. Here, we report on a female with a more classical BFLS phenotype and the first reported case of a large intragenic PHF6 deletion affecting several exons.

\section{Case Report}

The female patient was the first child of healthy, unrelated parents. She was born by Caesarian section due to placental insufficiency in gestation week 37. Her birth weight was $2.1 \mathrm{~kg}$, which was similar to her 2 healthy younger siblings. After an unremark- able neonatal period, she started to walk at age 1.5 and to speak a few words at age 3.5. Her intellectual deficits are compatible with mild mental retardation, but her IQ has never been tested. Behavior has been unremarkable. An EEG at age 7 showed increased theta activity, but she has never had any epileptic seizures. As a child she had recurrent middle ear infections, and now she suffers from mild hearing loss and uses bilateral hearing aids. Due to pigmented skin lines and bodily asymmetry, a mosaic condition of some kind was considered to be the most likely cause of developmental delay. At age 16, her height was $166 \mathrm{~cm}$ (50th centile) and her head circumference was $56.5 \mathrm{~cm}$ (75th centile), and at age 20, her weight was $64 \mathrm{~kg}$ (90th centile adjusted for height). Dysmorphic features and other findings are summarized in table 1 and illustrated in figure 1. Of note, her ear length was normal $(6 \mathrm{~cm}$, 50 th centile), and she also had irregular dentition, short roots, and hypodontia. Pigmented skin lines were best seen in the axillae (fig. 1c). Bodily asymmetry (larger left breast, $2 \mathrm{~cm}$ greater circumference of the left thigh, shorter left index finger) was also present. The prominent supraorbital ridges, hearing loss, and teeth abnormalities could indicate a skeletal dysplasia. However, the skeletal X-ray was normal apart from large frontal and maxillar sinuses and weak striations in the distal femur. A cerebral MRI at age 16 showed slightly widened ventricles and increased white 

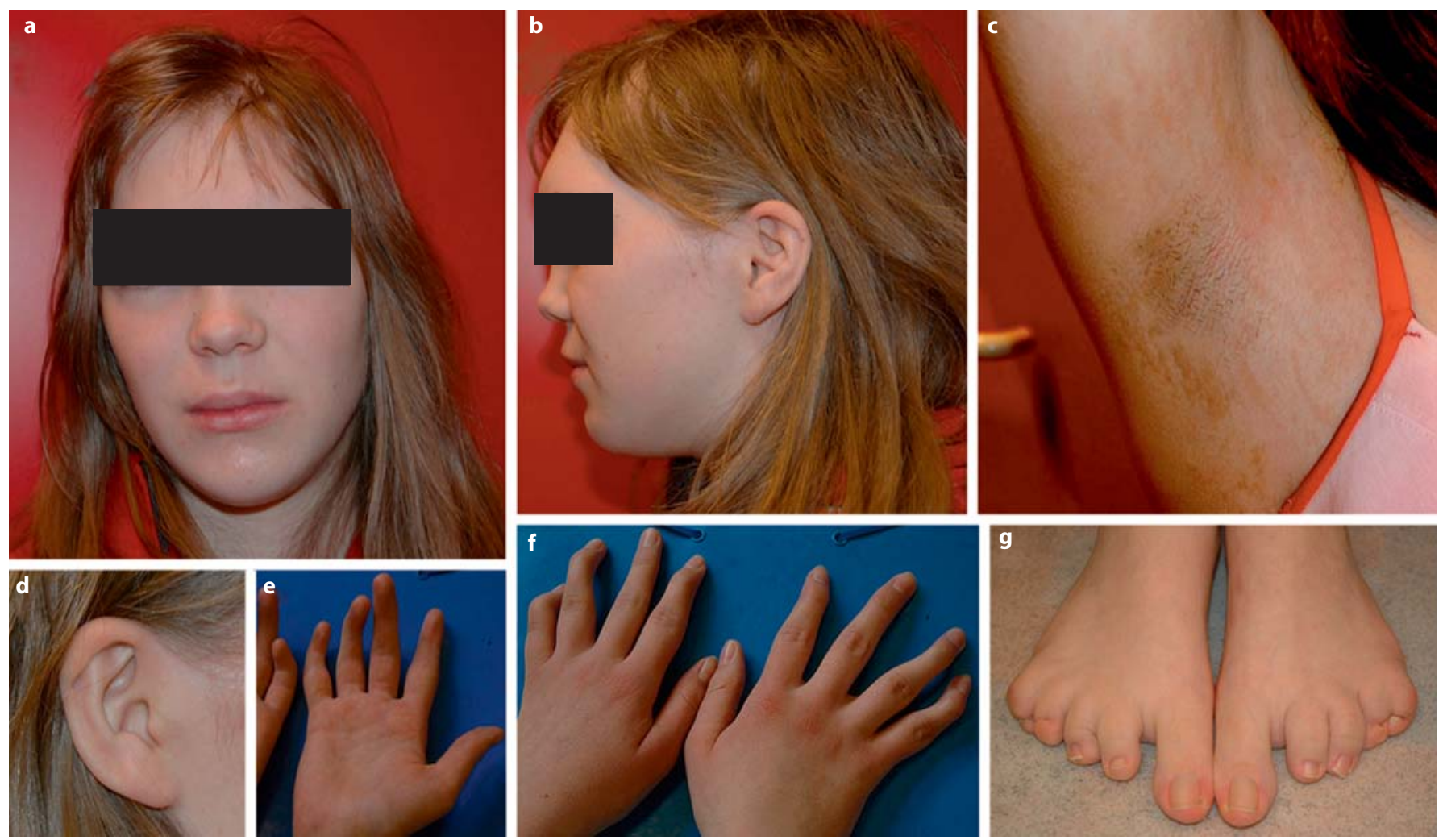

Fig. 1. Pictures of some clinical features. a Frontal view demonstrates an oval face with bitemporal narrowing, short nose with a bifid and squared tip, anteverted nares, thickened alae nasi, and a prominent chin. Palpebral fissures (not shown) are down slanting with hooded eyelids and hypertelorism. b Profile view demonstrates the secondary sloping forehead, scant frontal head hair, and high nasal ridge. c Right axilla with linear hyperpigmentation; slightly enhanced contrast for better illustration. $\mathbf{d}$ The ears have normal length and location, but the upper parts of the ears are short. The terminal portion of the crurae helices is expanded, and the descending helices are poorly marked and with slightly thickened lobes. e, f Slender and mallable fingers, and particularly the middle phalanges, show marked tapering. In addition, clino- and camptodactyly, short distal phalanges, distal flexion deformities, and shorter left 5 th finger are present. $g$ Her feet show short 2 nd -5 th toes, complete $4-5$ syndactyly bilaterally and partial 2-3 syndactyly on the left, hypoplastic $4-5$ th toenails, and a wide sandal gap. matter signaling of unspecific nature. Menarche occurred at age 14.5 with subsequent oligomenorrhea, with menstruation limited to a few times a year. LH and FSH levels were low with normal TSH and estrogens, compatible with mild hypogonadotropic hypogonadism.

\section{Methods}

DNA was purified from peripheral blood using QiaSymphony.

\section{X-Inactivation Studies}

A polymorphic trinucleotide repeat located in the first exon of the androgen receptor gene on chromosome Xq11.2-q12 was amplified using primers described in Allen et al. [1992]. The allele sizes of the trio were determined by amplification of untreated DNA from the parents, whereas the X-inactivation pattern in the daughter was determined by amplifying both un- treated DNA and DNA digested with methylation sensitive HpaII endonuclease. The labeled PCR products were separated according to size using capillary electrophoresis (ABI PRISM 3100 Genetic analyser).

Copy Number Analysis

High resolution copy number analysis was performed using Affymetrix Genome-Wide Human SNP Array 6.0. DNA prehandling and array hybridization was performed according to the manufacturer's instruction (Affymetrix) and scanned in an Affymetrix GeneChip Scanner 3000. Quality control, genotype calling, probe level normalization, and copy number normalization to produce $\log 2$ ratios were performed using Affymetrix GeneChip ${ }^{\circledR}$ Genotyping Console v3.0.1. The in-house-reference file was generated from 44 healthy blood donors. Data analysis and visualization were performed in Chromosome Analysis Suite with a threshold of $15 \mathrm{~kb}$ and 5 markers. Aberration is reported according to ISCN 2009 nomenclature and NCBI build 36. 


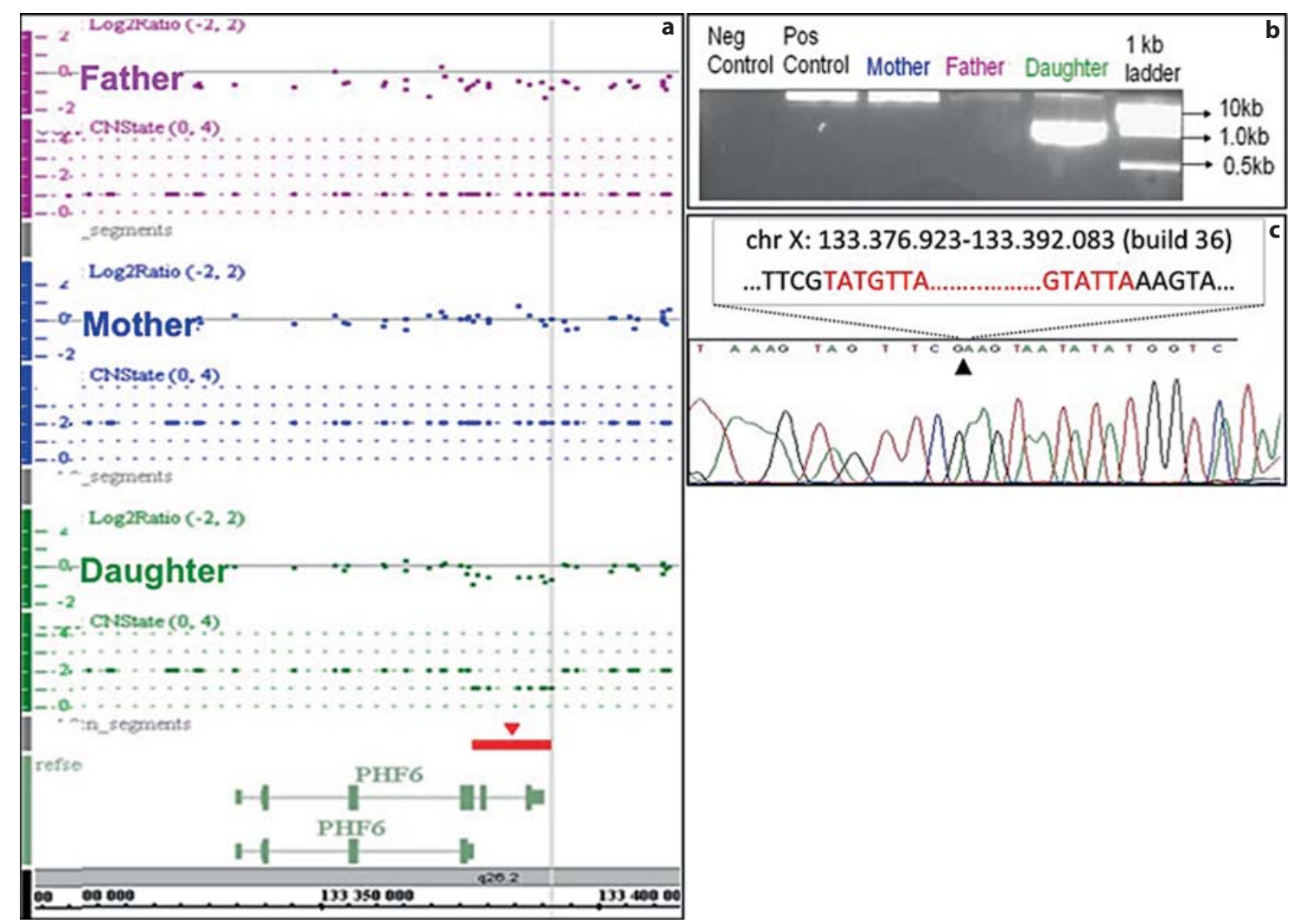

Fig. 2. a Affymetrix Genome-Wide Human SNP Array 6.0 showed a deletion involving the PFH6 gene on chromosome Xq26.3 (green). The mutation was de novo, given by the normal sequence of the patient's father (purple, $\mathrm{CN}$-state 1) and mother (blue, $\mathrm{CN}$ state 2 ). Genes in the region are illustrated below the proband's sequence. $\mathbf{b}$ Based on data from the 6.0 array, primers were placed in the flanking area and used for long template PCR. By separation on agarose gel, a normal product (size $16 \mathrm{~kb}$ ) was found in the

\section{Mapping the Breakpoint}

For mapping the breakpoint, the last normal and first aberrant markers on each site of the deletion were placed in the genomic contig NT_011786.15, and the primers were placed in between. The region was amplified using expand long template PCR system kit (Roche) with the primer pair PHF6-3F: 5'-AGTACATCCCAAATTTATCCAGTCA-3' and PHF6-3R: 5'-CTTGAACAACATTACAAGGTGTCAG-3', ELT PCR buffer 3, annealing temperature of $56^{\circ} \mathrm{C}$, extension of $14 \mathrm{~min}$, and a total of 35 cycles. PCR products were separated on an agarose gel.

\section{Genotyping}

The SNP information from the 6.0 SNP array was used for genotyping. The genotype was visualized using allelic difference, and by comparing the trio, SNP_8406469 was found informative. In order to sequence the parents across this SNP, new primers were designed and the PHF6 primer pair 5F: 5'-CAGTCACCTGCATGTCTGAAA-3' and 5R: 5'-ACATTCTTTCTGGACGTCCCT-3' was used for amplification with standard conditions for mother and father, with a weaker band in the daughter. The major product in the daughter was of approximately $1 \mathrm{~kb}$. c Sequencing the PCR product from the daughter identified a 15,160 bp deletion corresponding to basepairs 133.376.923-133.392.083 on the X chromosome (NCBI build 36). Black arrow: the exact location of the chromosomal break point; red letters: deleted nucleotides from the break point; black letters: normal nucleotides flanking the breakpoint.

AmpliTaq Gold. Combining the 5R primer with the 3F (above), the deleted allele in the daughter was amplified.

\section{Sequencing}

The PCR product from above was purified using ExoSAP-IT, and direct sequencing was performed using the ABI BigDye ${ }^{\circledR}$ Terminator v1.1 Cycle Sequencing Kit (Applied Biosystems) and ABI PRISM 3730 Genetic Analyser. The sequences were analysed using SeqScape ${ }^{\circledR}$ software v2.5.

\section{Results}

As chromosomal mosaicism was hypothesized based on the clinical findings of the patient, conventional Gbanded analysis of 30 metaphases from peripheral blood T-lymphocytes and 50 metaphases from skin fibroblasts 


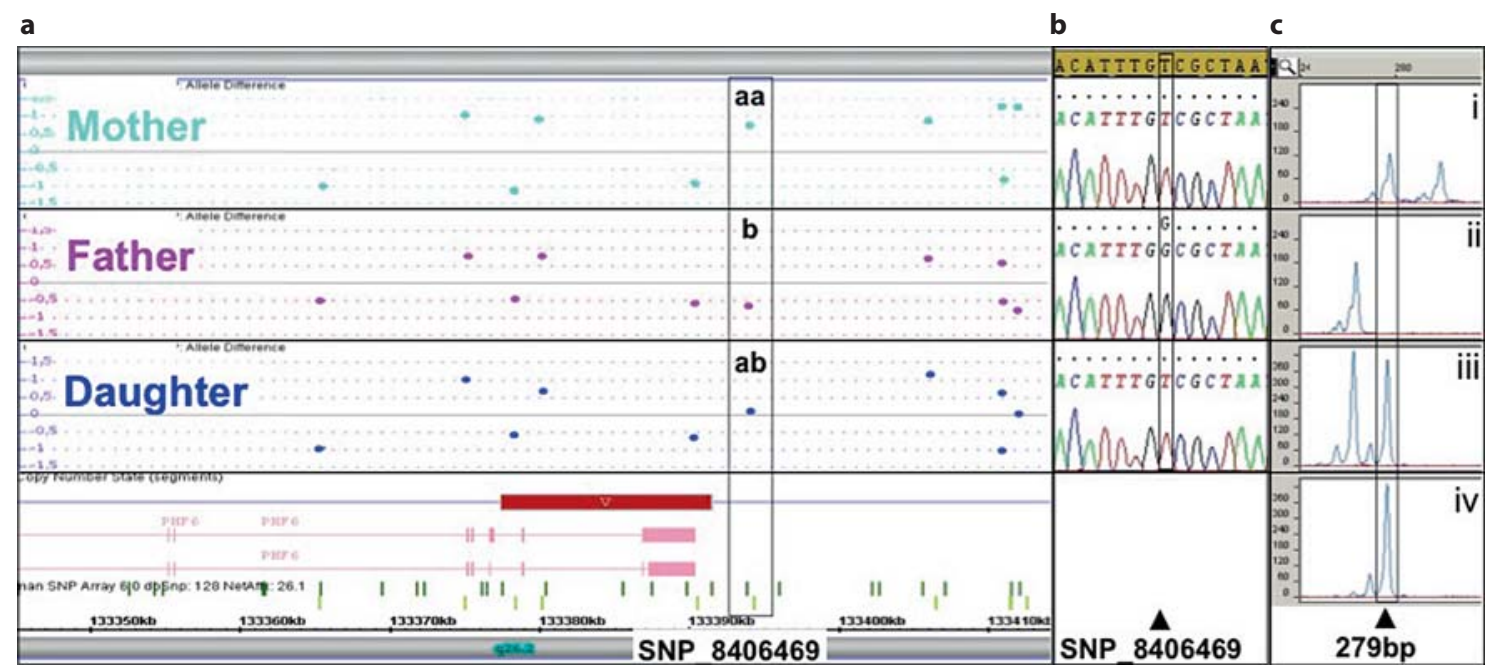

Fig. 3. a The genotype is visualized graphically by allelic difference. Dot at $+1=a a, 0=a b$, and $-1=b b$. By comparing the trio, we found no informative SNPs within the deletion, but the flanking SNP_8406469 appeared informative. b In order to get the genotype of SNP_840649 for the parents, new primers flanking this SNP were designed. For the daughter, the reverse primer from this design was used together with the forward primer used for mapping of the deletion, and this primer combination mainly amplified the deleted strand. The deleted allele of the daughter is of maternal origin. c Determination of the parental X-inactivation pattern was done by microsatellite fragment analysis of the $A R$ gene in position Xq11.2-q12. The polymorphic microsatellite in the $A R$ gene was found to be informative as the mother's 2 alleles (i, $279 \mathrm{bp}$ and $288 \mathrm{bp}$ ) are of different sizes than the father's (ii, 273 bp). The daughter has inherited the 279-bp allele from her mother and the 273-bp allele from her father (iii). The X-inactivation pattern of the daughter was determined by digesting patient DNA with methylation sensitive HpaII endonuclease prior to PCR. The 279-bp allele is present after cutting (iv), and thus the inactivated $\mathrm{X}$ chromosome is of maternal origin. were performed showing normal results. The abnormal skin pigmentation and bone striations could also indicate a functional mosaicism for an X-linked disease [Happle, 2006]. A standard $A R$ gene CAG-repeat X-inactivation test was performed [Allen et al., 1992], and the allelic ratio was highly skewed (96/4) after methylation-sensitive restriction enzyme digestion. One X-linked condition affecting females that could explain the supraorbital prominence, digital abnormalities, and hearing loss was otopalatodigital syndrome/frontometaphyseal dysplasia. However, sequencing of the filamin A gene, FLNA, gave normal results. When high-resolution genomic oligonucleotide arrays became available (Affymetrix GenomeWide Human SNP Array 6.0), a 14-19 kb deletion on Xq26.31 was found, with no indication of a mosaicism (fig. 2). The deletion was confirmed by long template PCR, and subsequent sequencing determined the deletion size to be $15,160 \mathrm{bp}$ without flanking rearrangements. The deletion starts in intron 8-9 and removes the final 3 exons (9-11) of the longest PHF6 transcript (ENST00000370803), including the second PHD domain. After investigating parental DNA samples, including an informative SNP near PHF6, the deletion was found to be de novo and of maternal origin (fig. 3). Furthermore, the mutated PHF6 allele was on the same $\mathrm{X}$ chromosome as the preferentially inactivated $A R$ allele (fig. 3). Her karyotype was 46,XX.arr Xq26.31 $(133,376,923-133,392,083) \times 1 \mathrm{dn}$ (NCBI build 36).

Loss-of-function mutations in PHF6 have recently been reported in T-cell acute lymphoblastic leukemia ( $\mathrm{T}$ ALL) [Van Vlierberghe et al., 2010]. Therefore, we wanted to see whether the mutated PHF6 gave T-lymphocytes a growth advantage. However, the differential leukocyte count of peripheral blood showed a normal cell distribution, and there was no measurable change in the $\mathrm{X}$-inactivation ratio after 6 days of PHA-stimulated cultivation of T-lymphocytes, which caused a doubling of the CD45 positive T-lymphocyte fraction.

\section{Discussion}

This is the first report of a large deletion in PHF6 causing BFLS, and it is of special interest as the patient is female. In the DECIPHER database, there are records of 2 females with copy number variants affecting PHF6, one 
$\sim 6-\mathrm{kb}$ intragenic deletion and one 1.17-Mb deletion including PHF6, but both are without clinical data. There are no known copy number variants involving PHF6 (see e.g. the Toronto Database of Genomic Variants, DGV), and patient 4630 reported in ECARUCA is the patient described in this paper.

Among the PHF6 mutations found in males with BFLS, 8 of 12 are missense mutations, 1 in-frame deletion, and 1 splice mutation. One of the 2 truncating mutations (R342X) is probably not affecting the PHF6-3 splice variant. This suggests that some residual PHF6 function (i.e. a hypomorphic mutation) is required for successful embryonic development. The deletion found in our patient removes the 3 terminal exons, including PHD domain 2, which most likely results in complete loss of PHF6 protein function (a null-allele). This is also the case for the only other female BFLS patient with a de novo early frame-shift mutation (G10fsX21) in PHF6 [Crawford et al., 2006]. In the literature, we have found female PHF6 mutation carriers with mild mental retardation (not only learning difficulties) in 3 families: in the original R342X family [Lower et al., 2004], in the Lys8X-family [Birrell et al., 2003; Turner et al., 2004], and in the family with a G89V missense mutation in the PDH-like domain 1 [Mangelsdorf et al., 2009]. This suggests that for PHF6 mutations to manifest as severe learning difficulties/ mental retardation or full-blown BFLS in females, more complete loss-of-function mutations are required. The most severe mutations might be incompatible with male fetal survival. In females, the phenotype is likely to be ameliorated by a skewed X-inactivation. Both our patient and the G10fsX21 girl showed a highly skewed X-inactivation in the blood with the mutated X chromosome being preferentially inactivated. In most BFLS families, female carriers have a skewed X-inactivation which is highly distinct ( $>90 \%)$ in about $50 \%$ of them [Lower et al., 2002; Turner et al., 2004; Crawford et al., 2006]. The vast majority of the females carrying a missense mutation, which are most probably hypomorphic, have no or only mild symptoms. This suggests that developmental aberrations caused by the PHF6 missense mutation are usually sufficiently compensated by a skewed $\mathrm{X}$-inactivation in most females.

We do not know why our patient has a near full-blown BFLS phenotype in spite of her favorable skewing of $\mathrm{X}$ inactivation. Compound heterozygosity can not be excluded as we have not sequenced both PHF6 alleles, but this option is considered unlikely. Some genes escape $\mathrm{X}$ inactivation but this appears not to be the case for PHF6, at least not in T-ALL [Van Vlierberghe et al., 2011]. The
X-inactivation pattern of $96 \%$ found in the blood is probably not representative for other organs and tissues, and sufficiently protective skewing might not be present in the brain, for example.

Our patient's features resemble male-type BFLS, i.e. mental retardation, prominent supraorbital ridges, deepset eyes, short and upturned nose, tapered fingers, hypermobility, short toes with syndactyly, and endocrine abnormalities (in our case hypogonadotrophic hypogonadism) [Visootsak et al., 2004; Carter et al., 2009] (table 1). Unlike most male patients, our patient also had dental abnormalities, hearing loss, and normally sized ears. Hearing impairment and dental anomalies have rarely been reported [Baumstark et al., 2003; Gecz et al., 2006; Carter et al., 2009]. Some other listed features such as severe mental retardation, short stature, microcephaly, and seizures also appear to be rare. Most of the features correlate well with the PHF6 gene expression pattern in mice [Voss et al., 2007] which was also prominent in the olfactory bulbs. Since the ability to smell was not tested in our patient, we do not know if anosmia could be a feature of this syndrome.

It is worth noting that female BFLS may mimic $2 \mathrm{X}$ linked disorders with skeletal dysplasia, namely frontometaphyseal dysplasia (MIM\#305620) and osteopathia striata with cranial sclerosis (MIM\#303373). Both diagnoses were suggested after the patient was presented at national and international syndrome meetings. As observed in osteopathia striata, longitudinal striations in the femoral epiphyses were also found in our patient (data not shown). Other signs of functional X-linked mosaicism were pigmented skin striations following Blaschko's lines and body asymmetry (fig. 1).

Finally, it should be noted that loss-of-function mutations in PHF6 have been reported in T-ALL or acute myeloblastic leukemia (AML), occurring predominantly in males [Van Vlierberghe et al., 2010, 2011]. In total, $70 \%$ of leukemia-mutations are nonsense or frame-shift. The missense mutations found have mostly been in the second PHD-like domain, i.e. exactly the same domain that was deleted in our patient (fig. 2). This strengthens our suspicion that loss of this domain may be detrimental to gene function. The loss-of-function mutations found in leukemias indicate that PHF6 is a tumor suppressor. However, it is not clear if the hypomorphic mutations found in the majority of the BFLS patients may be cancer predisposing. Among the few BFLS patients reported, 2 patients have been reported to have cancer. One male with a R257G missense developed Hodgkin's lymphoma at the age of 16 . Mutation at R257 is found in 
T-ALL. The other male diagnosed with T-ALL at age 7 carried the recurrent R342X non-sense mutation [Carter et al., 2009; Chao et al., 2010], a mutation also found in the AML sample [Van Vlierberghe et al., 2011]. We have not found signs of leukemia or lymphoma in our patient, but this is a risk that should be kept in mind during patient follow-up.

In conclusion, this case report underlines that BFLS should be considered among the possible diagnoses in females with developmental delay, signs of mosaicism, and a skewed X-inactivation pattern. The likelihood of a full-blown BFLS phenotype in females probably depends on both the genotype and the pattern of X chromosome inactivation.

\section{Acknowledgements}

We are most grateful to the patient and her family, whose enthusiastic collaboration made our work possible. We would also like to acknowledge the excellent work done by our genetic diagnostic laboratory, lead by Kjetil Solland, and peripheral blood Tcell analysis, done by Einar Kristoffersen.

\section{References}

-Allen RC, Zoghbi HY, Moseley AB, Rosenblatt HM, Belmont JW: Methylation of HpaII and HhaI sites near the polymorphic CAG repeat in the human androgen-receptor gene correlates with $\mathrm{X}$ chromosome inactivation. Am J Hum Genet 51:1229-1239 (1992).

-Ardinger HH, Hanson JW, Zellweger HU: Borjeson-Forssman-Lehmann syndrome: further delineation in five cases. Am J Med Genet 19: 653-664 (1984).

Baumstark A, Lower KM, Sinkus A, Andriuskeviciute I, Jurkeniene L, et al: Novel PHF6 mutation p.D333del causes Borjeson-Forssman-Lehmann syndrome. J Med Genet 40:e50 (2003).

-Birrell G, Lampe A, Richmond S, Bruce SN, Gecz $\mathrm{J}$, et al: Borjeson-Forssman-Lehmann syndrome and multiple pituitary hormone deficiency. J Pediatr Endocrinol Metab 16:12951300 (2003).

-Borjeson M, Forssman H, Lehmann O: An Xlinked, recessively inherited syndrome characterized by grave mental deficiency, epilepsy, and endocrine disorder. Acta Med Scand 171:13-21 (1962).

Carter MT, Picketts DJ, Hunter AG, Graham GE: Further clinical delineation of the BorjesonForssman-Lehmann syndrome in patients with PHF6 mutations. Am J Med Genet A 149:246-250 (2009).
Chao MM, Todd MA, Kontny U, Neas K, Sullivan MJ, et al: T-cell acute lymphoblastic leukemia in association with Borjeson-Forssman-Lehmann syndrome due to a mutation in PHF6. Pediatr Blood Cancer 55:722-724 (2010).

Crawford J, Lower KM, Hennekam RC, Van Esch H, Megarbane A, et al: Mutation screening in Borjeson-Forssman-Lehmann syndrome: identification of a novel de novo PHF6 mutation in a female patient. J Med Genet 43:238-243 (2006).

Dephoure N, Zhou C, Villen J, Beausoleil SA, Bakalarski CE, et al: A quantitative atlas of mitotic phosphorylation. Proc Natl Acad Sci USA 105:10762-10767 (2008).

de Winter CF, van Dijk F, Stolker JJ, Hennekam RC: Behavioural phenotype in BorjesonForssman-Lehmann syndrome. J Intellect Disabil Res 53:319-328 (2009).

- Gecz J, Turner G, Nelson J, Partington M: The Borjeson-Forssman-Lehman syndrome (BFLS, MIM \#301900). Eur J Hum Genet 14: 1233-1237 (2006).

Happle R: X-chromosome inactivation: role in skin disease expression. Acta Paediatr Suppl 451:16-23 (2006).

- Lower KM, Turner G, Kerr BA, Mathews KD, Shaw MA, et al: Mutations in PHF6 are associated with Borjeson-Forssman-Lehmann syndrome. Nat Genet 32:661-665 (2002).

- Lower KM, Solders G, Bondeson ML, Nelson J, Brun A, et al: 1024C $>$ T (R342X) is a recurrent PHF6 mutation also found in the original Borjeson-Forssman-Lehmann syndrome family. Eur J Hum Genet 12:787-789 (2004).
Mangelsdorf M, Chevrier E, Mustonen A, Picketts DJ: Borjeson-Forssman-Lehmann syndrome due to a novel plant homeodomain zinc finger mutation in the PHF6 gene. J Child Neurol 24:610-614 (2009).

- Turner G, Lower KM, White SM, Delatycki M, Lampe AK, et al: The clinical picture of the Borjeson-Forssman-Lehmann syndrome in males and heterozygous females with PHF6 mutations. Clin Genet 65:226-232 (2004).

-Vallee D, Chevrier E, Graham GE, Lazzaro MA, Lavigne PA, et al: A novel PHF6 mutation results in enhanced exon skipping and mild Borjeson-Forssman-Lehmann syndrome. J Med Genet 41:778-783 (2004).

-Van Vlierberghe P, Palomero T, Khiabanian H, Van der Meulen J, Castillo M, et al: PHF6 mutations in T-cell acute lymphoblastic leukemia. Nat Genet 42:338-342 (2010).

-Van Vlierberghe P, Patel J, Abdel-Wahab O, Lobry $\mathrm{C}$, Hedvat $\mathrm{CV}$, et al: PHF6 mutations in adult acute myeloid leukemia. Leukemia 25: 130-134 (2011).

-Visootsak J, Rosner B, Dykens E, Schwartz C, Hahn K, et al: Clinical and behavioral features of patients with Borjeson-ForssmanLehmann syndrome with mutations in PHF6. J Pediatr 145:819-825 (2004).

-Voss AK, Gamble R, Collin C, Shoubridge C, Corbett $\mathrm{M}$, et al: Protein and gene expression analysis of PhfG, the gene mutated in the Borjeson-Forssman-Lehmann Syndrome of intellectual disability and obesity. Gene Expr Patterns 7:858-871 (2007). 